increase with pressure so that, if rates permit, ECL will be formed with increasing abundance.

We thank Professor T. Evans for the use of his highpressure facilities. One of us (D. V. R.) acknowledges the receipt of a postgraduate award from the Science Research Council.

J. J. Thomson Physical Laboratory,

D. V. REES

D. C. BASSETT

University of Reading.

Received June 4; revised June $20,1968$.

Anderson, F. R., J. Appl. Phys., 35, 64 (1964).

${ }^{2}$ Wunderlich, B., and Arakawa, 'T., J. Polymer Sci., A, 2, 3697 (1964),

${ }^{3}$ Geil, P. H.. Anderson, F'. R., Wunderlich, B., and Arakawa, T., J. Polymer Sici., A, 2, 3707 (1964).

${ }^{4}$ Statton, W. O., and Geil, P. H., J. Appl. Polymer Sci., 3, 357 (1960).

'Hoffman, J. D., and Weeks, J. J., J. Chem. Phys., 42, 4301 (1965).

"Wunderlich, B., J. Polymer Sci., B, 5, 7 (1967).

Keller, A, and O'Connor, A., Nature, 180, 1289 (1957)

Balta Calleja, F. J., Bassett, D. C., and Keller, A., Polymer, 4, 269 (1963).

${ }^{9}$ Baer, E., and Kardos, J. L., J. Polymer Sei., A, 3, 2827 (1965).

\section{Charge Distributions of Conjugated Molecules}

PoINT charges located at the atom centres of conjugated molecules can be calculated using the Del Re procedure for $\sigma$ electrons, the Hückel procedure for $\pi$ electrons and the principle of $\sigma-\pi$ additivity. Such point charges, calculated by this and other procedures, have been used in biophysical problems ${ }^{1.1}$. Can the very simple Del ReHückel procedure give reasonable values for the charges ? This report provides a partial answer by comparing observed dipole moments with those calculated from De Re-Hückel charges obtained with established sets of tho nccessary parameters ${ }^{5,6}$.

The most appropriate comparison is with vapour phase dipole moments of molecules of known vapour phase structure. In five of the twenty-two compounds found to satisfy these criteria the components of the dipole moment along the principal molecular axes were available and were used as an added test of the computed charges. The comparisons are presented in Table 1 and the parameters used are given in Table 2 . The bromine $\sigma$ parameters are new and were determined by fitting the dipole moments of $\mathrm{CH}_{3} \mathrm{Br}$ and $\mathrm{CHBr}_{3}$ (refs. 7 and 8 ). We observed (Table 1 ) i:nprovement in the calculated moments of carbonyl-

Table 1. COMPARLSOY OF CALCULATED AND ORSERVED DIPOLE MOMENTS

$$
\text { Compound }
$$

Chlorobenzene 7,8

m-Dichlorobenzente

$\mu_{A}^{9}$
$\mu^{9}$ $\mu_{\mathrm{B}}{ }^{{ }^{a}}$

Fluorobenzene $e^{7,8}$

$m$ - Difluorobenzene

Fluoroethylene?
$\mu_{\mathrm{s}}^{7,10}$ $\mu_{\mathrm{i}}{ }^{7,10}$
$\mu_{\mathrm{B}}{ }^{7,10}$

1,1-Difluoroethylewe $e^{7,8}$ is-diffuoroethylene 7.8

p-Bromofluoroben Bromobenzene

'Toluene $e^{7,8}$

Pyridinc 7.8

Pyridinc 78

Pyrrolet?

Formaldehyde 7,8

Formaldeh

Acetone

ehyre, $\mu_{A}{ }^{11}$

i-Aerolein 12$$
\mu_{\mathrm{A}}{ }^{12}
$$

Formic acid ${ }^{19,14}$ acid 13,14 $\mu_{\mathrm{A}}{ }^{13.14}$
$\mu_{\mathrm{B}}{ }^{13.14}$ -Dichlorobenzene $e^{7,8}$

Cis-dichloroethylene $e^{7,8}$

$$
\text { Calculated (D) }
$$

$\mu_{\pi}$
$0 \cdot 29$
$0 \cdot 49$
$0 \cdot 28$
$0 \cdot 30$
$0 \cdot 29$
$0 \cdot 07$
$0 \cdot 22$
$0 \cdot 51$
$0 \cdot 51$
$0 \cdot 55$
$0 \cdot 54$
$0 \cdot 13$
$1 \cdot 04$
$0 \cdot 29$
$0 \cdot 29$
$0 \cdot 22$
$0 \cdot 23$
$0 \cdot 82$
$1 \cdot 12$
$2 \cdot 42$
$1 \cdot 94$
$\cdot 60(1 \cdot 41)$
$\cdot 10(2 \cdot 26)$
$\cdot 50(1 \cdot 89)$
$3 \cdot 20(1 \cdot 71)$
$\cdot 42(0 \cdot 79)$
$4.70(2 \cdot 18)$
$\cdot 57(2 \cdot 09)$
$\cdot 11(0 \cdot 64)$
$3 \cdot 76(2 \cdot 04)$
$3 \cdot 50(1 \cdot 89)$
$1 \cdot 38(0 \cdot 77)$

Observed

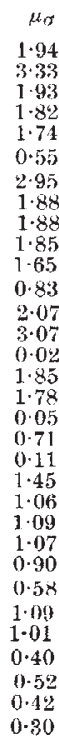

$\mu_{\text {total }}$
$1 \cdot 65$
$2 \cdot 84$
$1 \cdot 65$
1.58
$1 \cdot 45$
$0 \cdot 63$
$2 \cdot 73$
$1 \cdot 37$
$1 \cdot 37$
$1 \cdot 48$
$1 \cdot 12$
$0 \cdot 97$
$1 \cdot 03$
$6 \cdot 78$
$0 \cdot 27$
$1 \cdot 63$
$1 \cdot 59$
$0 \cdot 77$
$1 \cdot 83$
$2 \cdot 53$
$0 \cdot 49$
$3.66(2 \cdot 47)$
$5 \cdot 19(3 \cdot 35)$
$4 \cdot 56(2 \cdot 95)$
$4 \cdot 10(2 \cdot 61)$
$2 \cdot 00(1 \cdot 37)$
$5 \cdot 78(3 \cdot 28)$
$5.58(3 \cdot 10)$
$1 \cdot 52(1 \cdot 04)$
$3 \cdot 26(1 \cdot 54)$
$3 \cdot 08(1 \cdot 47)$
$1 \cdot 08(0 \cdot 47)$

$\mu$

$1 \cdot 70$

$2 \cdot 64$
$1 \cdot 68$

1.45

1.42

1.89

1.61
1.58
1.43
1.28

1.28

0.63

$1 \cdot 37$

$2 \cdot 42$

$1 \cdot 70$

1.42

$0 \cdot 35$
$2 \cdot 15$

1.84
0.66

$2 \cdot 34$

$2 \cdot 34$
2.90

$2 \cdot 90$
$2 \cdot 69$

2.55
0.87

3.11

$3 \cdot 11$
3.06

0.54

$1 \cdot 35$

$1 \cdot 33$
$0 \cdot 21$
Table 2. Parameter values for $\sigma$ and $\pi$ CAlqulations

\begin{tabular}{|c|c|c|c|c|c|c|c|c|c|}
\hline d & $\mathbf{C}^{\prime}-\mathrm{H}$ & $c-\mathrm{C}$ & $-x$ & $\mathrm{C}-\mathrm{O}$ & $\mathrm{N}-\mathrm{H}$ & $\mathbf{O}-\mathbf{H}$ & $\mathrm{C}-\mathrm{F}$ & $\mathrm{C}-\mathrm{Cl}$ & $\mathrm{C}-\mathrm{Br}$ \\
\hline & $1 \cdot 4$ & & & & & 0.4 & 5 & 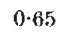 & $0 \cdot 60$ \\
\hline 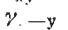 & $0 \cdot 30$ & 0 & 0.1 & & 0.8 & 0.3 & 0.1 & 0 & 0.20 \\
\hline & 0.40 & 0.1 & 0.10 & 0.1 & 0.4 & 0. & 0. & 0 & 0.40 \\
\hline & 0.0 & 0.07 & 0.0 & & & & & & 0.07 \\
\hline & 0.00 & 0.07 & 0.24 & 0.40 & 0.00 & 0.00 & 0.57 & $0-35$ & 0.30 \\
\hline
\end{tabular}
$\sigma^{*}$

Bond $\mathrm{C}-\mathrm{C}\left(\mathrm{C}-\ddot{\mathrm{N}}<\mathrm{C}-\dot{\mathrm{N}}=\mathrm{C}-\mathrm{O}-\mathrm{C}-\dot{\mathrm{O}} \mathrm{C}-\mathrm{C}_{3} \quad \mathrm{C}-\mathrm{F} \quad \mathrm{C}-\mathrm{Cl} \quad \mathrm{C}-\mathrm{Br}\right.$

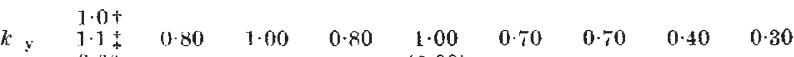

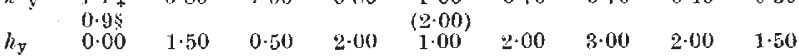

* Charges computed using the formula for bond charges as in ref. 15.

+ For bond length of about 1.40 A.

$\$$ For bond length of about $1.34 \AA$.

$\$$ For bond length of about $1 \cdot 47 \AA$.

containing compounds (values in brackets in Table 1) when the value of $k_{\mathrm{C}=0}$ was changed from $1 \cdot 0^{6}$ to $2 \cdot 0$, another generally used value ${ }^{16-19}$.

This communication extends recent evaluations ${ }^{20,21}$ of the Del Re-Hückel type calculations of charge distributions to include dipole moment components and previously unexamined heteroatoms, as well as a larger numerical sample. The agreement between computed and observed moments emphasizes that this simplistic procedure gives reasonable values for the point charges.

We thank Mrs Ann-Marie Reardon for programming and performing many of the computations in this work.

H. A. NASH

S. R. Grossman

D. F. Bradley

Laboratory of Neurochemistry,

National Institute of Mental Health,

Bethesda, Maryland.

Received June 13, 1968.

${ }^{2}$ Bradley, D. F., Lifson, S., and Honig, B., Electronic Aspects of Biochemistry (edit. by Pullman, B.), 77 (Acader. Be, Blectr, 1064).

${ }^{2}$ Nash, H. A., and Bradley, D. F., Biopolymers, 3, 261. (1965).

${ }^{3}$ Nash, H. A., and Bradley, D. F., J. Chem. Phys., 45, 1380 (1968).

4 Bradley, D. F. and Nash, H. A., Molecular Associations in Biology (ecit. by Pullman, B.), 137 (Academic Press, 1968).

'Del Re, G., J. Chem. Soc., 4031 (1968).

'Streitwieser, jun., A., Molecular Orbital Theory for Organic Chemists (Wilcy, 1961).

${ }^{7}$ Sutton, L. E., Tables of Interatomic Distances and Configurations in Molecules and Ions, Special Publication No. 18 of The Chemical Society, London (1965).

${ }^{8}$ McClellan, A. L., Tables of Experimental Dipole Moments (W. H. H'reeman, San Francisco, 1963).

Kivelson, D., Wilson, jun., E. B., and Lide, jun., D. R., J. Chem. Phys., $32,205(1960)$.

${ }^{10}$ Mirri, A. M., Guarnieri, A., and Favero, P., N. Cimento, 19, $1189(1961)$.

1 Kilb, R. W. Lin, C. C., and Wilson, jun., E. B., J. Chem. Phys, 26, 1695 (1957).

${ }^{12}$ Wagner, R., Fine, J., Simmons, J. W., and Goldstein, J. H., J. Chem. Phys., 26,634 (1957).

${ }^{13}$ Lerner, R. G., Dailey, B. P., and Friend, J. P., J. Chem. Phys., 26, 680 (1957).

${ }^{14}$ Erlandsson, G., and Selen, H., Ark. Fys., 14, 61 (1958).

${ }^{15}$ Del Re, G., Electronie Asperts of Biochemistry (edit. by Pullman, B.), 221 (Academic Press, 1964).

18 Coulson, C. A Trans. Fared Soc., 42, 106 (1964).

17 Berthier, G., Pullman, B., and Pontis, J., J. Chim. Phys., 49, 367 (1952). ${ }_{18}$ Goodwin, T. H., J. Chem. Soc., 1684 (1955).

${ }^{9}$ DeVoe, H. J., and Tinoco, jun., 1., J. Mol. Biol., 4, 500 (1062).

go Kier, L, B, Tetrahedron Lett, 37, 3273 (1965).

" Berthod, H., and Pullman, A., J. Chim. Phys., 62, 942 (1965).

\section{Benzo[a]pyrene, Phenols and other Products from Pyrolysis of the Cigarette Additive, (d,I)-Menthol}

ThIs communication surveys the chief products from the thermal degradation of $(d, l)$-menthol and discusses some qualitative and quantitative differences in product composition at two selected temperatures. The thermal decomposition of menthol-a naturally occurring alcohol 